\title{
Blood Pressure Home Monitoring in Hypertensive Patients Attending a Tertiary Health Facility in Amman, Jordan: Effect on Disease Control and Adherence Rate
}

\author{
Needa A Zalloum ${ }^{1}$, Rana Abu Farha ${ }^{1}$, Ala' M Abu Ruqa'a ${ }^{2}$, Ayman A Khdair ${ }^{3}$ and \\ Iman A Basheti ${ }^{4 *}$ \\ ${ }^{1}$ Department of Biopharmaceutics and Clinical Pharmacy, Faculty of Pharmacy, University of Jordan, ${ }^{2}$ Hayat Pharmaceutical \\ Industries, Co. Plc, Amman, ${ }^{3}$ Department of Pharmacy, Faculty of Health Sciences, American University of Madaba, Madaba, \\ ${ }^{4}$ Department of Clinical Pharmacy \& Therapeutics, Faculty of Pharmacy, Applied Science University, Amman, Jordan
}

*For correspondence: Email: dr_iman@asu.edu.jo; Tel: +962 799048 003; Fax: +9626 5515017

\begin{abstract}
Purpose: To investigate the effect of home monitoring of blood pressure (HMBP) on adherence to antihypertensive medications and control of the disease among patients attending a tertiary health facility in Amman, Jordan.

Methods: This cross-sectional correlation study was conducted in 2012 at the cardiovascular clinics of Jordan University Hospital. A questionnaire investigating participants' disease level of control, disease duration, frequency of HMBP use, type of measuring devices used, and effect of HMBP on adherence to antihypertensive medications and life style changes was used for the study. To minimize any bias that may have resulted from the involvement of pharmacists in data collection, we designed a clear stringent protocol that was strictly followed by the researcher with all patients. In addition to that, the researcher clearly introduced herself as an independent figure that cannot make any decisions or changes.

Results: Two hundred and five hypertensive patients who practice HMBP were recruited into this study. Sixty percent of the patients were aged 50 - 69 years. About $45 \%$ of the patients were diagnosed with hypertension for $>10$ years. Almost half of the patients have been using digital devices for blood pressure monitoring. The mean rate of HMBP was 15 times per month but almost $80 \%$ of the patients did not document their blood pressure readings. Around $55 \%$ of the patients referred high readings to a healthcare professional. Patients with higher levels of education as well as patients with lower BP readings showed significantly higher rates of adherence to their antihypertensive medications. Patients with high BP readings reported that they exercise more and reduced their daily salt intake.

Conclusion: Overall, the patients showed high adherence rates to antihypertensive medications and adoption of better life style. However, increasing patient's awareness in respect to documenting and communicating BP readings to their physicians is needed.
\end{abstract}

Keywords: Adherence, Hypertension, Compliance, Blood pressure monitoring, Salt intake, Antihypertensive, Jordan

Tropical Journal of Pharmaceutical Research is indexed by Science Citation Index (SciSearch), Scopus, International Pharmaceutical Abstract, Chemical Abstracts, Embase, Index Copernicus, EBSCO, African Index Medicus, JournalSeek, Journal Citation Reports/Science Edition, Directory of Open Access Journals (DOAJ), African Journal Online, Bioline International, Open-J-Gate and Pharmacy Abstracts

\section{INTRODUCTION}

Hypertension is a crucial worldwide public-health problem that is considered one of the most significant risk factors for cardiovascular diseases [1,2]. Despite the decline in heart disease over the last 30 years, it remains the leading cause of death around the world $[3,4]$. It 
has been predicted that by the year 2025, more than 1.5 billion individuals worldwide will have hypertension, accounting for up to $50 \%$ of heart disease risk and $75 \%$ of stroke risk [5].

In spite of the clear advantages of controlling hypertension in reducing cardiovascular morbidity and mortality, and regardless of the availability of many efficacious drug and nondrug treatments, blood pressure control among populations has not reached any ideal rate [1]. Physicians' suboptimal role in treating uncontrolled blood pressure, adverse effects of blood pressure lowering drugs, attitudes of patients in relation to disease and treatments, and lack of structured and efficient health care facilities are some reasons for the under management of hypertension $[6,7]$.

In Jordan, the prevalence of hypertension is very high, approaching $16 \%$ [8]. Amongst this population of hypertensive patients, it was found that only $30 \%$ of the patients had controlled blood pressure (BP) values, with the majority of patients not achieving their blood pressure goals [8].

Many factors have been identified to significantly affect blood pressure control [6-10]. Amongst these factors, non compliance to therapy was found to be a major cause of in the undermanagement of this chronic condition. Among all patient groups, non-compliance has long been the primary concern of both medical and pharmaceutical professionals as it has been associated with treatment failure, uncontrolled hypertension and disease progression $[11,12]$.

Home monitoring of blood pressure (HMBP) has become interestingly a popular method used to assess patient's blood pressure control. It has become more accepted and used by patients and physicians, since it is associated with less observer bias and no white coat effects. The higher number of readings obtained through HMBP correlate better with daytime values $[13,14]$, it is less expensive as a tool, and is able to detect patients with masked hypertension (blood pressure that is higher outside of the health professional's office) [15]. For this purpose, there are currently numerous appropriate devices available which have been rigorously tested. However, in Jordan, little is known about patients' perspectives of these devices and its effect on adherence.

The primary aim of this study is to investigate the effect of HMBP on adherence rates in an out- patient Jordanian population previously diagnosed with hypertension.

\section{EXPERIMENTAL}

\section{Patients and study design}

This cross-sectional correlation study design was conducted in 2012 for three months (April to July). During the study period, patients above the age of 18 years, with diagnosis of primary hypertension, receiving antihypertensive treatment (for at least 6 months) and using a HMBP were invited to participate in this study. Exclusion criteria include presence of any mental disorder or being involved in another clinical trial. Participants visiting cardiovascular clinics at Jordan University Hospital were approached for participation in the study. Participants who agreed to take part in the study provided informed oral consents.

The questionnaire was in two parts. The first part investigated participants' demographic information. The second part assessed the participants' health status such as diagnosis, level of control, disease duration, frequency of HMBP, type of the devices used, how the device was used (self-monitoring versus others performing the monitoring for the patient), effect of HMBP on adherence to antihypertensive medications, and an assessment of blood pressure control for the patient.

To ensure face validity, the questionnaire (in both English and Arabic languages) was evaluated by three academics who have a previous experience in conducting clinical studies and have a wide range of clinical professional experience. The questionnaire was then completed by pharmacy students $(n=15)$ to test for clarity of questions. Views and comments of the students were considered by the researchers and then incorporated where appropriate into the final version of the questionnaire. Completed questionnaires were inserted in envelops by the researcher and returned for data entry and analysis.

\section{Data analysis}

Participants' responses were encoded and the data were analyzed using Statistical Package for the Social Sciences (SPSS, version 17, Chicago, IL, US). Descriptive analysis was done using mean and SD for continuous variables and percentage for qualitative variables. Chi square test was used to identify any significant difference among the participants' responses with significance defined as $p$ value of $\leq 0.05$. 
Pearson's product-moment correlation was used to analyze relationships between continuous variables for within group comparisons.

Sample size calculation was based on the population of Amman (1,206,266 in the year 2010) [16], with $16 \%$ prevalence of hypertension $(193,002)$ [8]; a minimum sample size of 384 was calculated, for a margin error of $5 \%$, confidence level of $95 \%$, and a response distribution of 50 $\%$.

\section{RESULTS}

Three hundred and eighty five patients were approached to participate in the study during the study period. Two hundred and five participants agreed to participate. About half of the respondents (52.7\%) were females. Average age range of respondents was 23-85 year, with the majority (59\%) aged between 50 and 69 years. A smaller proportion (20.5\%) was in the age range of $20-49$ or above the age of 70 years. Only $9.8 \%$ of the participants were not educated, while the rest either completed secondary school (35\%), community college (12.6\%), Bachelor of Science degree (32.2\%), master degree $(7.1 \%)$ or Ph.D. degree (3.3\%).

Thirty percent of participants were employed, 36 $\%$ were unemployed, and $34 \%$ were retired, with the majority $(94.6 \%)$ reporting working status in a non-medical field.

A majority of participants were diagnosed with hypertension for less than 10 years, while others were hypertensive for longer periods (37.1\% between 10 and 20 years, and $8.3 \%$ for more than 20 years). When the participants were asked about the frequency of their follow up visits, many of them (30\%) reported visiting their specialist four times yearly (a visit every three months), while some (28\%) visited their doctor 12 times yearly, and the rest visited between 4 and 12 times a year.

Respondents reported measuring their blood pressure $15.5 \pm 7.83$ times a month and only a minority of the participants $(20.3 \%)$ reported recording their blood pressure readings.

Regarding the type of home measuring devices used by the participants, the majority (52\%) used the wrist digital devices, $44 \%$ the mercury based devices, and $4 \%$ the air-meter devices. About half of the respondents $(51.9 \%)$ were dependent on someone else to perform their blood pressure monitoring. Lower educational levels and not working status were associated significantly with this finding $(p<0.001)$. Significant association was found between age and type of device used, in that older age was significantly associated with the use of air meter or mercury meter than digital meter devices ( $r=$ $0.239, p=0.001$ Spearman's correlation).

Participants repeated their blood pressure measurements about 1.62 times on each occasion if their blood pressure was found high. Most of the respondents $(69.3 \%)$ reported not forgetting their antihypertensive medications, and $89.4 \%$ declared that they do not stop their antihypertensive medications in any circumstance, even if their blood pressure was within normal values $(86.7 \%)$. Some patients $(25.7 \%)$ reported stopping their antihypertensive treatment in the case of side-effects only.

Participants reported detecting low pressure readings about $4.1 \pm 1.04$ times a month, with the majority (52.8\%) responding with increasing their salt intake. A minority (17 \%) reported decreasing or skipping their medication dose.

As for high blood pressure values, participants reported detecting high levels around $3.1 \pm 1.2$ times a month, with $75.6 \%$ responding by cutting down on their salt levels, changing their diet or exercise (64.2 \%), or increasing their antihypertensive medication dose (26.3\%).

About half of the respondents (54.8\%) reported visiting the physician or the emergency room in response to abnormal blood pressure readings.

Response to high blood pressure values differed significantly due to different educational status, period of diagnosis, and working status $(P=$ $0.018, p=0.024$, and $p=0.005$ respectively).

There was a significant correlation between reporting higher frequency of blood pressure measuring and having more stabilized blood pressure readings $(r=-0.232, p=0.002$, Spearman's correlation).

Patients who reported using the digital meter change their antihypertensive medication in response to their blood pressure readings significantly more than people using the other two meters $(p=0.037)$.

Logistic regression analysis with "forgetting to take antihypertensive medication" as the dependent variable, reported significant association with patients' education and blood 
pressure results, in that higher education ( $p=$ $0.044)$ and low blood pressure readings $(p=$ 0.002) significantly correlated with better adherence (Table 1).

\section{DISCUSSION}

To our knowledge, this study is considered the first study to investigate the effect of HMBP on hypertensive patients' adherence to antihypertensive medications in Jordan. This study sheds light on important facts regarding the use of home self-monitoring blood pressure devices, which is a relatively new and emerging practice.

Results indicate a high frequency (15 times per month with about 2 repetitions for abnormal readings) of self-measuring of $B P$ in patients who own BP measuring devices. The importance of frequent blood pressure self-measuring has been acknowledged previously (17 - 20). Higher rates of self-monitoring have been associated with better prediction of stroke risk as compared to more frequent physician assessment in the clinics (20). Although Brook et al reported that the accuracy of HBPM does not depend on the number of measurements per day, Verberk et al recommended that two duplicates a day is the minimum number for effective self-monitoring $(13,21)$. Results from this study indicated that patients who measured their blood pressure more frequently, had a significantly better control over their hypertension. This finding is vital, as it can be used as a proof of the benefit of HMBP, paving the way towards better patient adherence to BP measuring and better control over disease. Although just over half of the respondents reported visiting a healthcare professional in response to abnormal BP readings, only $20 \%$ of patients reported recording BP measurements at home for later assessment of their disease management by their doctor. This indicated that more efforts need to be directed at making patients in Jordan better at utilizing data generated by HMBP through documented communication with their physician. Other studies have shown that HMBP patients in
Jordan are less adherent in recording their BP readings when compared to other countries, such as Canada (35.9\%) and China (73\%) $(15,22)$. The importance of reliable and accurate reporting of HBPM results to healthcare professional by all hypertensive patients has been stressed previously (23).

The higher proportion of patients using digital devices compared to the mercury based devices could be due to the simplicity of using these devices. Furthermore, users of digital devices reported a significantly higher rate of changes in their antihypertensive medication use than users of other types of devices. The high benefit of using electronic devices in controlling and reaching the target $\mathrm{BP}$ level has been previously established (24). On the other hand, the high percentage of mercury-based devices users, especially among elderly patients, could be linked to the familiarity of patients with these older devices in Jordan. Digital devices are considered a relatively new technology that has been introduced into the Jordanian market during the past couple of decades. The use of mercury devices explains why half of our patients rely on assistance (someone else) to perform their HMBP.

Patients' reported compliance with their antihypertensive medications was high in this study, with majority of the respondent reporting not stopping their medications due to their blood pressure self-assessment. This important outcome supports previous findings, in that adherence with antihypertensive medications is one of the advantages resulting from the use of the BP home-devices (14). Eventually, this would lead to a reduction in the number of patient's visiting their physician for continuous disease management.

Another advantage for the HMBP noticed in this study is patient's adoption of a better life style and diet. Abnormal BP readings resulting from the self-assessment helped patients in adjusting

Table 1: Summary of the logistic regression model for the dependent variable 'forgetting to take antihypertensive medications', with its associated predictors, size and direction of the association $(n=205)$

\begin{tabular}{llcc}
\hline Associated predictor & Beta & T & $\boldsymbol{P}$-value \\
\hline How often do you find your blood & 0.287 & 3.114 & 0.002 \\
pressure low? & 0.188 & 2.044 & 0.044 \\
\hline
\end{tabular}

"Beta" is the standardized regression coefficient, "Beta" values with their " $p$ " values show whether each variable is making a statistically unique contribution to the model $(P<0.05)$ or not. The t-test given by the " $T$ " value, tests the significance of each coefficient, it can determine the relative importance of each variable in the model (useful predictors usually have " $t$ " values above 2 or below -2) 
their daily salt intake. This practice among HMBP Jordanians went parallel with the findings of Yamasue et al, where $17 \mathrm{mmol}$ daily decrease in salt intake resulted in a significant reduction in BP based on self-measured readings (25). Another advantage of practicing HMBP found in current study was that about $64 \%$ of the patients in current study exercised more as a response to high self-readings of BP.

It is important for patients with hypertension to consult their healthcare provider for help in the case of extreme abnormal blood pressure values. Results of this study were positive in this regard, where more than half of the users of selfdevices referred the matter of abnormal readings to their physician or to the emergency department. On the other hand, some of the patients in this study (17\% to $26.3 \%$ ) reported skipping, decreasing or increasing their antihypertensive medication doses based on their self-readings. This is an essential drawback for the use of these devices at home, and increasing patient's awareness in this area is vital.

In the world of hypertension, patient education and awareness is important for proper management of the disease (7). This study showed that patients who referred their abnormal readings of $\mathrm{BP}$ to a healthcare professional were those with higher level of education. These patients also reported more significant adherence to their medications. The lower readings of blood pressure reported by the highly educated patients in this study may be considered a consequence for being more compliant with their antihypertensive medications. Education is an important factor highlighting patients on the risk of complications of high $\mathrm{BP}$ readings thus encouraging them to better comply with their antihypertensive medications.

\section{Limitations of the study}

It should be mentioned that the role of the pharmacist should have been investigated in this study to give a clear complete picture in this area. This is especially important as the pharmacist is usually the healthcare professional who sees the patients last before they use the BP self-measuring devices. It would have been useful to extrapolate socio-economic factors in the analysis by questioning the respondents about their income; however, this was not possible due to the sensitivity of this topic to many patients residing in a low income country such as Jordan. Finally, although the study presents a satisfactory approach to research in the area of $\mathrm{HMBP}$, it is challenging to draw stringent conclusions based on the methodology chosen. Thus, a broader follow-up study will answer many of the questions left unanswered in this investigation. This includes, for example, the effect of enhanced compliance (based on HMBP practice) on the control of blood pressure using in-clinic readings and patients' records.

\section{CONCLUSION}

The practice of HMBP has several advantages in relation to control of $\mathrm{BP}$. The findings of this study indicate that uneducated patients require further counseling on the necessity to refer abnormal BP readings to a healthcare professional and the importance of not changing their anti-hypertensive medications based solely on home readings. In addition, all patients, regardless of their level of education, require training on proper documentation of their BP home-readings for future communication with their healthcare providers.

\section{REFERENCES}

1. Kearney PM, Whelton M, Reynolds K, Whelton PK, He J. Worldwide prevalence of hypertension: a systematic review. J Hypertens 2004; 22: 11-19.

2. Wong N, Dede J, Chow V, Wong K. Franklin S. Global Cardiovascular Risk Associated With Hypertension and Extent of Treatment and Control According to Risk Group. AM J HYPERTENS 2012; 25: 561-567.

3. Burt VL, Whelton P, Roccella EJ, Brown C, Cutler JA, Higgins M, Horan MJ, Labarthe, D. Prevalence of hypertension in the U.S. population: results from the third national health and nutrition examination survey, 1988-1991. Hypertension 1995; 25: 305-313.

4. Chobanian AV, Bakris GL, Black HR, Cushman WC, Green LA, Izzo JL, Jones DW, Materson BJ, Oparil S, Wright JT, et al. Seventh Report of the Joint National Committee on Prevention, Detection, Evaluation, and Treatment of High Blood Pressure. Hypertension 2003; 42: $1206-1252$.

5. Kearney PM, Whelton M, Reynolds $K$, Muntner $P$, Whelton PK, He J. Global burden of hypertension: analysis of worldwide data. Lancet. 2005; 365(9455): 217-223.

6. Oliveria SA, Lapuerta $P$, McCarthy $B D$, L'ltalien GJ, Berlowitz DR, Asch SM. Physician-related barriers to the effective management of uncontrolled hypertension. Arch Intern Med 2002; 162: 413- 420.

7. Neutel JM, Smith DHG. Improving patient compliance: a major goal in the management of hypertension. J Clin Hypertens 2003; 5: 127-132. 
8. Jaddou HY, Bateiha AM, Ajlouni KM. Prevalence, awareness and management of hypertension in a recently urbanized community, eastern Jordan. J Hum Hypertens 2000; 14(8): 497-501.

9. Garcao JA, Cabrita J. Evaluation of a Pharmaceutical Care Program for Hypertensive Patients in Rural Portugal. J Am Pharm Assoc 2002; 42: 858-864.

10. Orozco-Beltrán D, Brotons $C$, Moral I, Soriano N, Valle $M$, Rodríguez A, Pepió J, Pastor A. Factors Affecting the Control of Blood Pressure and Lipid Levels in Patients With Cardiovascular Disease: The PREseAP Study. Rev Esp Cardiol. 2008; 61(3): 317-321.

11. SHEP Cooperative Research Group. Prevention of stroke by antihypertensive drug treatment in older persons with isolated systolic hypertension: final results of the systolic hypertension in the elderly program (SHEP). JAMA 1991; 265: 255-3264.

12. Rowland $M$, Roberts $M$. Blood pressure levels and hypertension in persons aged 6-74 years: United States, 1976-1980. Advance Data 1982; (84): 1-11.

13. Verberk WJ, Kroon A, Kessels A, de Leeuw PW. Home Blood Pressure Measurement: A Systematic Review. J Am Coll Cardiol 2005; 46 (5): 743-751.

14. Celis H, Den Hond E, Staessen J. FAHA. SelfMeasurement of Blood Pressure at Home in the Management of Hypertension. Clinical Medicine \& Research 2005; 3 (1): 19-26

15. Bancej C, Campbell N, McKay D, Nichol M, Walker R, Kaczorowski J. Home blood pressure monitoring among Canadian adults with hypertension: Results from the 2009 Survey on Living with Chronic Diseases in Canada. Can J Cardiol 2010; 26(5): e152-e157

16. Internet World States. [cited 2012 May 15]. Available from http://www. internetworldstats.com/.

17. Celis $H$, Singh $D$. Home blood pressure measurement appears useful for diagnosis and prognosis in routine clinical practice. Evid Based Cardiovasc Med. 2006; 10 (1): 18-20.

18. Cappuccio FP, Kerry SM, Forbes L, Donald A. Blood pressure control by home monitoring: meta-analysis of randomised trials. BMJ 2004; 329: 145.

19. Cushman WC, Duprez DA, Weintraub HS, Purkayastha $D$, Zappe D, Samuel R, Izzo L. Home and clinic blood pressure responses in elderly individuals with systolic hypertension. J Am Soc Hypertens 2012; 6 (3): 210218.

20. Ohkubo T, Asayama K, Kikuya M. How many times should blood pressure be measured at home for better prediction of stroke risk? Ten-year follow-up results from the Ohasama study. J Hypertens 2004; 22 2004:1099-1104

21. Brook RD. Home blood pressure accuracy is independent of monitoring schedules. Am J Hypertens 2000; 13 2000: 625-631.

22. Wang Y1, Wang Y, Gu H, Qain Y, Zhang J, Tang X, Sun $J$, Zhu D. Use of home blood pressure monitoring among hypertensive adults in primary care: Minhang community survey. Blood Press Monit. 2014; 19(3):140-4.

23. Mengden T, Hernandez Medina RM, Beltran B, Alvarez $E$, Kraft $K$, Vetter $H$. Reliability of reporting selfmeasured blood pressure values by hypertensive patients. Am J Hypertens. 1998; 11(12): 1413-7.

24. Santschi V, Rodondi N, Bugnon O, Burnier M. Impact of electronic monitoring of drug adherence on blood pressure control in primary care: A cluster 12-month randomised controlled study. Eur J Intern Med 2008; 19(6): 427-434.

25. Yamasue K, Tochikubo O, Kono E, Maeda H. Selfmonitoring of home blood pressure with estimation of daily salt intake using a new electrical device. $J$ Hum Hypertens. 2006; 20: 593-598. 